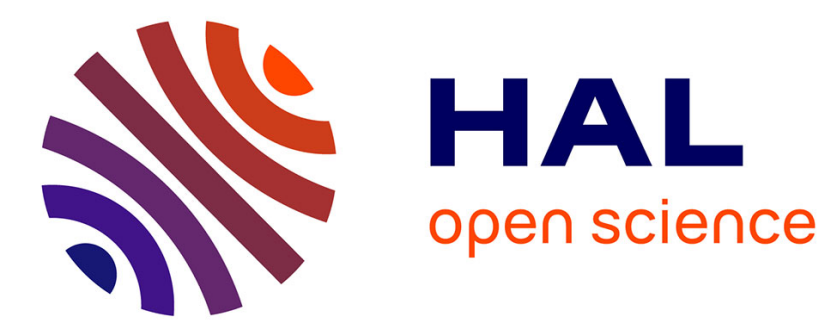

\title{
Power control in Wireless Cellular Networks with a time-varying delay
}

Hamed Samie, Emmanuel Moulay, Patrick Coirault, Rodolphe Vauzelle

\section{To cite this version:}

Hamed Samie, Emmanuel Moulay, Patrick Coirault, Rodolphe Vauzelle. Power control in Wireless Cellular Networks with a time-varying delay. Automatica, 2017, 83, pp.179 - 185. 10.1016/j.automatica.2017.06.034 . hal-01724035

\section{HAL Id: hal-01724035 https://hal.science/hal-01724035}

Submitted on 19 Jan 2021

HAL is a multi-disciplinary open access archive for the deposit and dissemination of scientific research documents, whether they are published or not. The documents may come from teaching and research institutions in France or abroad, or from public or private research centers.
L'archive ouverte pluridisciplinaire HAL, est destinée au dépôt et à la diffusion de documents scientifiques de niveau recherche, publiés ou non, émanant des établissements d'enseignement et de recherche français ou étrangers, des laboratoires publics ou privés. 


\title{
Power Control in Wireless Cellular Networks with a Time-Varying Delay
}

\author{
Hamed Samie $^{\mathrm{a}}$, Emmanuel Moulay ${ }^{\mathrm{b}}$, Patrick Coirault ${ }^{\mathrm{a}}$, Rodolphe Vauzelle ${ }^{\mathrm{b}}$ \\ ${ }^{\mathrm{a}} L I A S$ (EA 6315), 2 rue Pierre Brousse, 86073, Poitiers, France \\ ${ }^{\mathrm{b}}$ XLIM (UMR CNRS 7252), 11 Boulevard Marie et Pierre Curie, 86962, Futuroscope Chasseneuil, France
}

\begin{abstract}
Uplink transmission power control is an essential task in Wireless Cellular Networks (WCNs) due to the resource limitation of the Mobile Stations (MSs). One remaining problem is the effect of the delay caused by measuring the signal strength and decision making in the Inner-Loop Power Control (ILPC). In this article, we develop the Potential Feedback Controller (PFC) for a linear scalar discrete-time system with disturbance in order to take into account an unknown bounded time-varying input delay for uplink ILPC. The main interest of the PFC is to treat easily a stabilization problem with a constraint on the state space by using a nonlinear feedback control with a short computation time. Simulations illustrate that by applying the PFC, the communication connectivity is ensured by maintaining the signal strength above a required limit.
\end{abstract}

Key words: Power control; Potential feedback control; Time-varying delay; Wireless Cellular Networks.

\section{Introduction}

Power control in WCNs is a key degree of freedom in the management of interference, energy and connectivity [20]. In uplink transmission, energy efficiency aspect of power control is more dominant due to the limited energy resource of the Mobile Station (MS). Uplink power control in WCNs includes two main objectives $[24,23,17]$ :

- firstly, to ensure the communication connectivity by setting up a power controller to keep the received signal strength above a limit below which the communication is interrupted.

- secondly, to minimize the overall transmitted power in a cell in order to minimize the interferences between users and to maximize the battery life of the MS.

Principally, power control in WCNs consists of an open-loop power control and a closed-loop power con-

Email addresses: hamed.samie@univ-poitiers.fr (Hamed Samie), emmanuel.moulay@univ-poitiers.fr (Emmanuel Moulay),

patrick.coirault@univ-poitiers.fr (Patrick Coirault), rodolphe.vauzelle@univ-poitiers.fr (Rodolphe Vauzelle). trol schemes [20]. In open-loop power control, the Base Station (BS) selects the transmit power control by exploiting the estimated channel condition like distancedependent attenuation and frequency-independent slow fading at the MS based on the received signal strength of a pilot signal transmitted by the BS [15].

The closed-loop power control itself consists of two different loops: Inner-Loop Power Control (ILPC) and OuterLoop Power Control (OLPC) [10,15]. In ILPC, the received signal strength is compared to a target value at the BS. If the measured signal strength is higher (lower) than the target value, the BS will send a control signal to the MS in order to decrease (increase) the transmission power. The ILPC should be repeated fast enough to cover the fast fading effect. The OLPC (which is slower than the ILPC) provides the target signal strength based on the Quality of Service (QoS) requirements in the higher layers [15]. In this paper, we are interested in the ILPC.

Transmitting and measuring signals and decision making take time which results in time delays in the closedloop power control. The main reasons of these delays come from the power control algorithm itself; time for computing and decision making; time to transmit the power control command to the MS $[13,16,20]$. According to the time-varying behavior of the transmission channel 
quality, load of the MSs and the size of the packets to be sent in a realistic scenario, a time-varying delay in the closed-loop power control must be considered. The delay in uplink ILPC is discussed further in Section 2. Like every feedback control loop, the ILPC will be affected by this time-varying delay. In fact, the power control is more sensitive to the delay than to the signal strength estimator [10].

Stability of the OLPC in the presence of communication delays has been studied in $[22,7,14]$. In [10], the authors proposed a control scheme to highlight the dynamical behavior of the ILPC subject to a constant delay where they proposed a time-delay compensation (TDC) in order to mitigate the oscillations due to the delay. In our paper, we develop the PFC first proposed in [8] in order to take into account an unknown bounded time-varying input delay for uplink ILPC in WCNs. To the best of our knowledge, this is the first time that a solution for the time-varying delay in the ILPC of WCNs has been proposed.

The rest of the paper is organized as follows. The system model is described in Section 2. In order to take into account a time-varying input delay, the PFC is developed in Section 3. It is applied and simulated for a WCN using the Long-Term Evolution (LTE) communication standard and compared with the existing Transmission Power Control (TPC) algorithm in Section 4. Finally, a conclusion is addressed in Section 5.

\section{$2 \quad$ Modeling}

In our article, we are interested in the uplink ILPC of a cell with a MS denoted by $k$ and a BS denoted by $i$ which is illustrated in Figure 1. The received Signal-to-

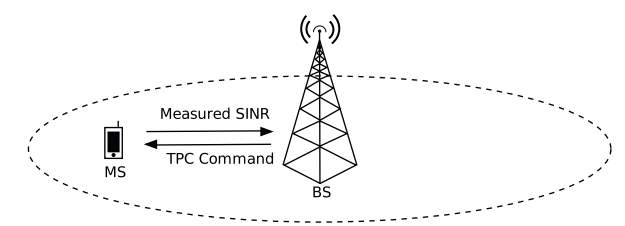

Fig. 1. Uplink ILPC between a MS and a BS

Interference-plus-Noise Ratio (SINR) is commonly used in wireless communication to measure the transmission quality. For simplicity, we neglect the intra-cell interferences (in communication standards like LTE and $802.11 n$, the use of respectively OFDMA and OFDM avoids the intra-cell interferences [11]). Therefore, after frequency synchronization, only the inter-cell interferences affect the SINR in the model. The received SINR at the BS $i$ of the transmitted signal from the MS $k$ is given by

$$
\chi_{i k}(n)=\frac{g_{i k}(n) p_{i k}(n)}{\sum_{l \neq k} g_{i l}(n) \cdot p_{i l}(n)+\sigma_{i k}^{2}(n)}
$$

where $p_{i k}$ is the transmitted power; $g_{i k}$ the channel gain by which the signal between the transmitter $k$ and the receiver $i$ is attenuated and it can be modeled by three components: path-loss, log-normal shadowing and multipath fading $[25] ; \sigma_{i k}^{2}$ the thermal noise affecting the channel between the MS $k$ and the BS $i ; n$ represents the ILPC sample time. The term $\sum_{l \neq k} g_{i l}(n) \cdot p_{i l}(n)$ corresponds to the inter-cell interferences. As it has been assumed that the intra-cell interferences are neglected, we can rewrite (1) as follows

$$
\chi_{i k}(n)=\frac{g_{i k}(n) \cdot p_{i k}(n)}{i_{i k}(n)+\sigma_{i k}^{2}(n)}
$$

where $i_{i k}(n)=\sum_{l \neq k} g_{i l}(n) \cdot p_{i l}(n)$ are the inter-cell interferences. Let us define the pathloss $p l$ between the MS $k$ and the BS $i$ by $p l_{i k}(n)=\frac{1}{g_{i k}(n)}$ and $i_{o} t_{i k}(n)=$ $\frac{\left(i_{i k}(n)+\sigma_{i k}^{2}(n)\right)}{\sigma_{i k}^{2}(n)}$. We can rewrite $(2)$ as follows

$$
\chi_{i k}(n)=\frac{p_{i k}(n)}{p l_{i k}(n) \cdot \sigma_{i k}^{2}(n) \cdot i_{o} t_{i k}(n)} .
$$

In the following, we rewrite the received SINR equation in the logarithmic domain in $\mathrm{dB}$. Consequently, equation (3) becomes

$$
x_{i k}(n)=P_{i k}(n)-P L_{i k}(n)-\Sigma_{i k}^{2}(n)-I_{o} T_{i k}(n) .
$$

\section{Uplink ILPC in WCNs}

In uplink ILPC, the SINR is estimated at the MS and it is sent to the BS where it will be compared with a SINR $_{\text {min }}$ which is the minimum required value of SINR to maintain the communication connectivity. Based on its difference with the SINR $_{\text {min }}$, a TPC command is sent to the MS to update the transmission power $[4,10,3]$. So we can describe this procedure as follows:

- Receiver (BS):

$$
\begin{aligned}
\operatorname{err}_{i}(n) & =x_{i k}(n)-x_{m i n} \\
u_{i}(n) & =f\left(\operatorname{err}_{i}(n)\right)
\end{aligned}
$$

where $x_{\text {min }}$ is the $\mathrm{SINR}_{\text {min }} ;$ err $r_{i}$ the difference between the available measured SINR at the sample time $n$ and the $\mathrm{SINR}_{\text {min }} ; u_{i}$ the TPC command computed at the BS to be sent to the MS which is a function of the $\mathrm{err}_{i}$ as it is explained above. The SINR $_{\text {min }}$ is provided by the OLPC at a lower update rate based on the required value of the Block Error Rate (BLER) in higher layers [20]. Hence, this value may be regarded constant for the ILPC. There exist different algorithms based on the different communication standards applied in WCNs $[4,10,3]$. For example in LTE, the TPC command $u_{i}$ can vary between $[-1 ; 3] \mathrm{dBm}$ (for more information see [4]). In Section 4, we will compare the PFC with taking into account an unknown bounded time-varying input delay with an existing TPC algorithm in LTE communication standard. - Transmitter (MS):

Evolution of the transmit power at the MS is given by the following equation

$$
P_{i k}(n+1)=P_{i k}(n)+u_{i}(n-\tau(n))
$$


where $P_{i k}$ is the transmit power adjustment. It should be taken into account that the computed uplink transmit power should not exceed the maximum MS transmit power $\mathrm{P}_{\max }[20,6]$. For simplicity, we do not take into account the $\mathrm{P}_{\max }$ in our calculation but we will take it into account in our simulations in Section 4.

Both measuring and signaling in cellular systems take time, which results in delayed signals. Here are the main reasons for the existense of time delay in power control update in WCNs [20]:

- the power control algorithm itself results in a delay of one sample time since the power control at sample time $n$ is used to update the power level at sample time $n+1$; - it takes certain amount of time to measure the SINR and generate a power control decision at the BS;

- the power control update is only allowed to be transmitted at certain time instants;

- the time that it takes to transmit the power control command from the BS to the MS.

As it has been explained in Section 1, this delay $\tau(n)$ must be considered as a time-varying delay. The capacity of the network and the details of the communication standard are known, therefore the maximum and minimum values of $\tau(n)$ are known too:

$$
0<h_{1} \leq \tau(n) \leq h_{2}
$$

where $h_{1}$ and $h_{2}$ are respectively the minimum and the maximum value of the time-varying delay.

In the following, and for simplicity, we will omit the subscripts $i$ and $k$. By putting (4) into the dynamical equation (6), we obtain

$$
\begin{aligned}
x(n+1) & =x(n)+u(n-\tau(n))+P L(n)+\Sigma^{2}(n) \\
& +I_{o} T(n)-P L(n+1)-\Sigma^{2}(n+1) \\
& -I_{o} T(n+1)
\end{aligned}
$$

Also, we have

$$
\begin{aligned}
P L(n+1) & =P L(n)+\Delta P L(n) \\
\Sigma^{2}(n+1) & =\Sigma^{2}(n)+\Delta \Sigma^{2}(n) \\
I_{o} T(n+1) & =I_{o} T(n)+\Delta I_{o} T(n)
\end{aligned}
$$

By putting (9) into (8), we obtain

$$
x(n+1)=x(n)+u(n-\tau(n))+d(n)
$$

where $d(n)=-\Delta P L(n)-\Delta \Sigma^{2}-\Delta I_{o} T(n)$. In the following section, we develop the PFC in order to take into account the time-varying input delay $\tau(n)$.

\section{PFC for discrete-time systems with a time- varying input delay}

In this section, we develop a new result on stabilization by using the Potential Feedback Controller (PFC) first proposed in [8], in order to take into account an unknown bounded time-varying input delay for the uplink ILPC of WCNs. Stability of linear discrete-time dynamical systems with an unknown bounded input delay has been discussed in several works. For example [2], [26] and [19] propose different solutions by means of Linear Matrix Inequalities (LMIs) and [28] gives a solution by designing a predictor-based sliding surface. The potential control has originally been applied in robotics to avoid collisions with obstacles [21]. The main idea of exploiting the potential control for the ILPC is to maintain the signal strength (SINR) higher than a target value SINR $_{\text {min }}$ defined by the OLPC while keeping the SINR as close as possible to the $\mathrm{SINR}_{\text {min }}$ in order to be more energy efficient. The proof of this result is based on the Artsein's reduction method proposed in [2].

Theorem 3.1 Consider the system given by

$$
x(n+1)=x(n)+u(n-\tau(n))+d_{1}(n)
$$

where $x(n) \in \mathbb{R}$ is the state of the system, $|u(n)| \leq M$ is the bounded input with $0<h_{1} \leq \tau(n) \leq h_{2} \in \mathbb{N}$ a bounded time-varying delay and $\left|d_{1}(n)\right| \leq \bar{\varepsilon}$ a bounded disturbance. Let $c \leq 0$, under the initial condition $x(0)>$ $c$, system (11) in closed-loop with the feedback control

$$
u(n)=k_{1}(z(n)-c)+\frac{k_{2}}{z(n)-c}
$$

where $u(n)=0$ for $n<0$ and

or

$$
\left\{\begin{array}{l}
-\frac{1}{2}<k_{1}<\frac{0.5\left(-h_{1}-h_{2}+1\right)}{h_{1}+h_{2}} \\
k_{2}>\frac{\left(k_{1}+1\right)(\varepsilon+2 M)^{2}}{\left(2 k_{1}+1\right)^{2}}
\end{array}\right.
$$

$$
\left\{\begin{array}{l}
\frac{0.5\left(-h_{1}-h_{2}+1\right)}{h_{1}+h_{2}}<k_{1}<0 \\
k_{2}>\frac{0.25(\varepsilon+2 M)^{2}\left(h_{1}+h_{2}+1\right)^{2}}{k_{1}+1}
\end{array}\right.
$$

and $z(n)=x(n)+\frac{1}{2} \sum_{j=0}^{h_{1}-1} u\left(n-h_{1}+j\right)+\frac{1}{2} \sum_{j=0}^{h_{2}-1} u\left(n-h_{2}+\right.$ $j)$ is asymptotically stable with respect to the attractive and invariant set $S=\left[\sqrt{4\left(k_{1}+1\right) k_{2}}-(\varepsilon+2 M)-\left(h_{1}+\right.\right.$ $\left.h_{2}\right)(\varepsilon+2 M)+c ; \frac{-(\varepsilon+2 M)\left(k_{1}-1\right)+\sqrt{(\varepsilon+2 M)^{2}\left(k_{1}-1\right)^{2}-4 k_{1}^{3} k_{2}}}{2 k_{1}^{2}}+$ $\left.(\varepsilon+2 M)+\left(h_{1}+h_{2}\right)(\varepsilon+2 M)+c\right]$. Moreover, we have $x(n) \geq \sqrt{4\left(k_{1}+1\right) k_{2}}-(\varepsilon+2 M)-\left(h_{1}+h_{2}\right)(\varepsilon+2 M)+c$ for all $n>h_{1}+h_{2}$.

Proof. We have two possible cases which depend on the sign of the initial condition $x(0)$ of system (11).

First case: $x(0)>0$. We take $c=0$. By applying the Artstein transform as explained in [2], we can transform system (11) into the following scalar discrete-time system without delay

$$
z(n+1)=z(n)+u(n)+d_{1}(n)+d_{2}(n)
$$

where $z(n)$ is obtained by the following transformation

$$
\left\{\begin{array}{l}
z(n)=x(n)+\phi_{n}^{u}\left(h_{1}\right)+\phi_{n}^{u}\left(h_{2}\right) \\
\phi_{n}^{u}(h)=\frac{1}{2} \sum_{j=0}^{h-1} u(n-h+j)
\end{array}\right.
$$

and $d_{2}(n)$ is an extra disturbance caused by applying the Artstein transform and given by the following equation

$$
d_{2}(n)=u(n-\tau(n))-\frac{1}{2}\left(u\left(n-h_{1}\right)+u\left(n-h_{2}\right)\right)
$$

A simple calculation based on bounded input assumption $|u(n)| \leq M$ shows that $\left|d_{2}\right| \leq 2 M$.

As it has been explained in [8, Theorem 1], system (13) 
with the feedback control

$$
u(n)=k_{1} z(n)+\frac{k_{2}}{z(n)}
$$

where $\frac{-1}{2}<k_{1}<0$ and $k_{2} \geq \frac{(\varepsilon+2 M)^{2}\left(k_{1}+1\right)}{\left(2 k_{1}+1\right)^{2}}$ is asymptotically stable with respect to the attractive set $S_{2}=\left[\sqrt{4\left(k_{1}+1\right) k_{2}}-(\varepsilon+2 M) ; \frac{-(\varepsilon+2 M)\left(k_{1}-1\right)}{2 k_{1}^{2}}+\right.$ $\left.\frac{\sqrt{(\varepsilon+2 M)^{2}\left(k_{1}-1\right)^{2}-4 k_{1}^{3} k_{2}}}{2 k_{1}^{2}}\right]$.

According to [8, Theorem 1], we have

$$
\min _{z(n) \in S_{2}} z(n+1) \geq \min S_{2} .
$$

Therefore, due to the variations of the continuous function $z(n+1)$ with respect to the variable $z(n)$ which is increasing and the Intermediate Value Theorem, we only have to prove that the following conditions

$$
\begin{aligned}
& \left.z(n+1)\right|_{z(n)=\min S_{2}} \leq \max S_{2} \\
& \left.z(n+1)\right|_{z(n)=\max S_{2}} \leq \max S_{2}
\end{aligned}
$$

where $\min S_{2}=\sqrt{4\left(k_{1}+1\right) k_{2}}-(\varepsilon+2 M)$ and $\max S_{2}=\frac{-(\varepsilon+2 M)\left(k_{1}-1\right)+\sqrt{(\varepsilon+2 M)^{2}\left(k_{1}-1\right)^{2}-4 k_{1}^{3} k_{2}}}{2 k_{1}^{2}}$, are satisfied in order that $S_{2}$ be an invariant set.

By applying the control feedback (16) to system (13), we obtain

$$
z(n+1)=\left(k_{1}+1\right) z(n)+\frac{k_{2}}{z(n)}+d_{1}(n)+d_{2}(n)
$$

Afterwards, by solving the equation $\frac{\partial z(n+1)}{\partial z(n)}=0$, we obtain the critical point $z_{c}=\sqrt{\frac{k_{2}}{k_{1}+1}}$. An investigation into the behavior of system (13) around $z_{c}$ shows that $z(n+1)$ is increasing on $\left[\sqrt{\frac{k_{2}}{k_{1}+1}},+\infty\right)$ and decreasing on $\left(0, \sqrt{\frac{k_{2}}{k_{1}+1}}\right.$. Therefore, $z_{c}$ is the minimum value of $z(n+1)$. We have

$$
\begin{aligned}
z_{\min } & =\left.z(n+1)\right|_{z(n)=z_{c}} \\
& =\left(1+k_{1}\right) \sqrt{\frac{k_{2}}{k_{1}+1}}+\frac{k_{2}}{\sqrt{\frac{k_{2}}{k_{1}+1}}}-(\varepsilon+2 M) \\
& =\sqrt{4\left(k_{1}+1\right) k_{2}}-(\varepsilon+2 M)=\min S_{2}
\end{aligned}
$$

Considering that $\left.z(n+1)\right|_{z(n)=z_{c}}=\min S_{2}$, and $z(n+1)$ is increasing on $\left[\sqrt{\frac{k_{2}}{k_{1}+1}},+\infty\right)$, we deduce that if $\min S_{2} \geq \sqrt{\frac{k_{2}}{k_{1}+1}}$ and the inequality (19) is fulfilled then inequality (18) is fulfilled too. First, we check that

$$
z_{\min }=\sqrt{4\left(k_{1}+1\right) k_{2}}-(\varepsilon+2 M) \geq \sqrt{\frac{k_{2}}{k_{1}+1}}
$$

which leads to

$$
\frac{\sqrt{k_{2}}\left(2 k_{1}+1\right)}{\sqrt{k_{1}+1}} \geq(\varepsilon+2 M)
$$

where $\sqrt{k_{2}} \geq 0$ and $\sqrt{k_{1}+1} \geq 0$. Thus, we have $-\frac{1}{2}<k_{1}<0$. Also from (22), we obtain $k_{2} \geq \frac{(\varepsilon+2 M)^{2}\left(k_{1}+1\right)}{\left(2 k_{1}+1\right)^{2}}$. Now it is sufficient to show that inequality (19) is fulfilled. We have

$$
\left.z(n+1)\right|_{z(n)=\max S_{2}}=\frac{N_{1}}{D}
$$

where

$$
\begin{aligned}
N_{1}= & \left(k_{1}+1\right)\left[-(\varepsilon+2 M)\left(k_{1}-1\right)\right. \\
+ & \sqrt{\left.(\varepsilon+2 M)^{2}\left(k_{1}-1\right)^{2}-4 k_{1}^{3} k_{2}\right]} \\
+ & 4 k_{1}^{4} k_{2}+2(\varepsilon+2 M) k_{1}^{2}\left(-(\varepsilon+2 M)\left(k_{1}-1\right)\right. \\
+ & \sqrt{\left.(\varepsilon+2 M)^{2}\left(k_{1}-1\right)^{2}-4 k_{1}^{3} k_{2}\right)} \\
D & =2 k_{1}^{2}\left(-(\varepsilon+2 M)\left(k_{1}-1\right)\right. \\
& +\sqrt{\left.(\varepsilon+2 M)^{2}\left(k_{1}-1\right)^{2}-4 k_{1}^{3} k_{2}\right) .}
\end{aligned}
$$

and

We can rewrite $\max S_{2}$ as follows

$$
\begin{aligned}
\max S_{2} & =\frac{\left(-(\varepsilon+2 M)\left(k_{1}-1\right)+\sqrt{(\varepsilon+2 M)^{2}\left(k_{1}-1\right)^{2}-4 k_{1}^{3} k_{2}}\right)^{2}}{2 k_{1}^{2}\left(-(\varepsilon+2 M)\left(k_{1}-1\right)+\sqrt{\left.(\varepsilon+2 M)^{2}\left(k_{1}-1\right)^{2}-4 k_{1}^{3} k_{2}\right)}\right.} \\
& =\frac{N_{2}}{D} .
\end{aligned}
$$

In order that inequality (19) be fulfilled, we must have $N_{2}>N_{1}$. Thus, a series of calculations lead to

$$
\begin{aligned}
& 2 k_{1}\left(k_{1}-1\right)(\varepsilon+2 M)^{2}- \\
& 2(\varepsilon+2 M) k_{1} \sqrt{(\varepsilon+2 M)^{2}\left(k_{1}-1\right)^{2}-4 k_{1}^{3} k_{2}}>0
\end{aligned}
$$

which is satisfied considering that $-\frac{1}{2}<k_{1}<0$. Hence, we conclude that $S_{2}=\left[\sqrt{4\left(k_{1}+1\right) k_{2}}-(\varepsilon+\right.$ $\left.2 M) ; \frac{-(\varepsilon+2 M)\left(k_{1}-1\right)}{2 k_{1}^{2}}+\frac{\sqrt{(\varepsilon+2 M)^{2}\left(k_{1}-1\right)^{2}-4 k_{1}^{3} k_{2}}}{2 k_{1}^{2}}\right]$ is an attractive and invariant set with $-\frac{1}{2}<k_{1}<0$ and $k_{2} \geq \frac{(\varepsilon+2 M)^{2}\left(k_{1}+1\right)}{\left(2 k_{1}+1\right)^{2}}$.

By applying the Artstein transform, we derive that system (11) is asymptotically stable with respect to a set $S$ we have to determine. From (13) we obtain

$$
\left\{\begin{array}{l}
\sum_{j=0}^{h_{1}-1} u\left(n-h_{1}+j\right)=\left[z(n)-z\left(n-h_{1}\right)-\right. \\
\left.\sum_{j=0}^{h_{1}-1} d_{1}\left(n-h_{1}+j\right)-\sum_{j=0}^{h_{1}-1} d_{2}\left(n-h_{1}+j\right)\right] \\
=2 \phi_{n}^{u}\left(h_{1}\right) \\
\sum_{j=0}^{h_{2}-1} u\left(n-h_{2}+j\right)=\left[z(n)-z\left(n-h_{2}\right)-\right. \\
\left.\sum_{j=0}^{h_{2}-1} d_{2}\left(n-h_{2}+j\right)-\sum_{j=0}^{h_{2}-1} d_{2}\left(n-h_{2}+j\right)\right] \\
=2 \phi_{n}^{u}\left(h_{2}\right) .
\end{array}\right.
$$

From (14) and (24) we have

$$
x(n)=\frac{1}{2}\left(z\left(n-h_{1}\right)+z\left(n-h_{2}\right)\right)+\gamma(n)+\lambda(n)
$$

where $\gamma(n)=-\frac{1}{2}\left[\sum_{j=0}^{h_{1}-1} d_{1}\left(n-h_{1}+j\right)+\sum_{j=0}^{h_{1}-1} d_{2}\left(n-h_{1}+j\right)\right]$ and $\lambda(n)=\frac{-1}{2}\left[\sum_{j=0}^{h_{2}-1} d_{1}\left(n-h_{2}+j\right)+\sum_{j=0}^{h_{2}-1} d_{2}\left(n-h_{2}+j\right)\right]$. As $z($.$) is bounded due to [8, Theorem 1] and d_{1}$ and $d_{2}$ are bounded by assumption, $x($.$) is also bounded due$ to (25). Accordingly, there exists a minimum value $x_{\min }$ such that the state $x($.$) of system (13) is always higher$ than $x_{\text {min }}$. From (25) we derive that

$$
x_{\min }=z_{\min }-\left(h_{1}+h_{2}\right)(\varepsilon+2 M)
$$

where $z_{\min }=\sqrt{4\left(k_{1}+1\right) k_{1}}-(\varepsilon+2 M)$ due to (21). This 
minimum value $x_{\min }$ is also the minimum value of the attractive and invariant set $S$. As $x_{\min }>0$, a symbolic calculation leads to

$$
\sqrt{4\left(k_{1}+1\right) k_{1}}-(\varepsilon+2 M)-\left(h_{1}+h_{2}\right)(\varepsilon+2 M)>0(27)
$$

In order to satisfy (27), we obtain the following sufficient conditions:

or

$$
\left\{\begin{array}{l}
-\frac{1}{2}<k_{1}<\frac{0.5\left(-h_{1}-h_{2}+1\right)}{h_{1}+h_{2}} \\
k_{2}>\frac{\left(k_{1}+1\right)(\varepsilon+2 M)^{2}}{\left(2 k_{1}+1\right)^{2}}
\end{array}\right.
$$

$$
\left\{\begin{array}{l}
\frac{0.5\left(-h_{1}-h_{2}+1\right)}{h_{1}+h_{2}}<k_{1}<0 \\
k_{2}>\frac{0.25(\varepsilon+2 M)^{2}\left(h_{1}+h_{2}+1\right)^{2}}{k_{1}+1}
\end{array}\right.
$$

with $\varepsilon>0,2 M>0, h_{2}>1$ and $h_{1}>0$.

Moreover, it is easy to check that the maximum value of $S$ satisfies

$$
\max S=\max S_{2}+\left(h_{1}+h_{2}\right)(\varepsilon+2 M) .
$$

So we have found the attractive and invariant set $S$. As we have $z(n)>z_{\min }$ for all $n>0$, then we have $x(n)>x_{\min }$ for all $n>h_{1}+h_{2}$.

Second case: $x(0)<0$. From (11) and (12) we have

$$
\begin{aligned}
x(n+1) & =x(n)+k_{1}(x(n-\tau(n))-c) \\
& +\frac{k_{2}}{x(n-\tau(n))-c}+d_{1}(n)
\end{aligned}
$$

By subtracting $c$ in both sides, we obtain

$$
\begin{aligned}
x(n+1)-c & =x(n)-c+k_{1}(x(n-\tau(n))-c) \\
& +\frac{k_{2}}{x(n-\tau(n))-c}+d_{1}(n) .
\end{aligned}
$$

Considering the following change of variable $\psi(n)=$ $x(n)-c$, it leads to the new system

$\psi(n+1)=\psi(n)+k_{1}(\psi(n-\tau(n)))+\frac{k_{1}}{\psi(n-\tau(n))}+d_{1}(n)$

As $x(0)>c$, we have $\psi(0)>0$, therefore, we can apply the first case of the proof to system (33).

Finally we deduce that if $x(0)>c, \varepsilon>0,2 M>0$, $h_{2}>1, h_{2} \geq \tau(n) \geq h_{1}>0$ and $k_{1}$ and $k_{2}$ are chosen by (28) or (29) then, system (11) is asymptotically stable with respect to the attractive and invariant set $S=\left[\sqrt{4\left(k_{1}+1\right) k_{2}}-(\varepsilon+2 M)-\left(h_{1}+h_{2}\right)(\varepsilon+2 M)+\right.$ $c ; \frac{-(\varepsilon+2 M)\left(k_{1}-1\right)}{2 k_{1}^{2}}+\frac{\sqrt{(\varepsilon+2 M)^{2}\left(k_{1}-1\right)^{2}-4 k_{1}^{3} k_{2}}}{2 k_{1}^{2}}+(\varepsilon+2 M)+$ $\left.\left(h_{1}+h_{2}\right)(\varepsilon+2 M)+c\right]$. This concludes the Proof.

\section{Simulation for the LTE standard}

The characteristics of the LTE communication standard such as mobility of the MSs which leads to a timevarying transmission channel quality and intense timevarying load of the MSs for each BS imply that the ILPC has a significant time-varying behavior involving a timevarying delay. Consequently, we chose the LTE communication standard as a typical example to illustrate the PFC with taking into account an unknown bounded time-varying input delay.

We suppose that there is an estimation error of $1 \mathrm{~dB}$ on the $d(n)$ in (10). The assessment of this value depends on the quality of the channel gain estimator (see [8]). For our simulations it has been assumed that the cell radius is $500 \mathrm{~m}$ as well as the distance between the MS and the BS which varies between $18.08 \mathrm{~m}$ and $342 \mathrm{~m}$ therefore, the $P L$ varies between $63.93 \mathrm{~dB}$ and $89.18 \mathrm{~dB}$.

As it has been described in Section $2, x_{\min }$ which represents the $\mathrm{SINR}_{\text {min }}$ is provided by the OLPC based on the highest acceptable BLER for communication between the transmitter and the receiver. The maximum BLER given by LTE communication standard is $10^{-1}$ [12]. In LTE, Adaptive Modulation and Coding (AMC) module based on the Channel Quality Indicator (CQI) index chooses a specific Modulation and Coding Scheme (MCS) and a Code Rate to transmit the data (for further explanation of the Physical and Medium Access Control (MAC) layers in LTE communication standard, the reader may refer to [5]). Therefore, based on the relation between the SINR and the BLER for a given MCS and Code Rate, we can define the $\mathrm{SINR}_{\min }$ [27]. For our simulations we choose the 64-QAM MCS with Code Rate $2 / 3$. Hence, we find the value of $\operatorname{SINR}_{\text {min }}=14.37 \mathrm{~dB}$ (for further details see [8]).

The coherence time of the channel is given by

$$
T_{c}=\sqrt{\frac{9}{16 \pi f_{\max }^{2}}}
$$

where $f_{\max }=\frac{v}{\lambda}$ is the maximum Doppler Shift with $v$ the velocity of the MS [9]. The control sample time $T_{s}$ at which we refresh the feedback control cannot exceed the coherence time of the channel. In LTE, the carrier frequency $f_{c}$ is equal to $1.8 \mathrm{GHz}$ where $f_{c}=\frac{c}{\lambda}$. By taking into account the various possible amounts of MS velocity up to $260 \mathrm{~km} . \mathrm{h}^{-1}$, we obtain the coherence time $T_{c}=1 \mathrm{~ms}$. Hence, we choose the sample time $T_{s}=1 \mathrm{~ms}$ which is a common value for the sample time in LTE communication standard [11].

In order to take into account the round-trip delay in LTE communication standard, the TPC algorithm takes the maximum round-trip delay as a constant delay. The maximum round-trip delay in LTE is $20 \mathrm{~ms}$ [13]. Thus, in our simulations we compare the performances of a TPC algorithm considering a maximum round-trip delay of $20 \mathrm{~ms}[4,18]$, the PFC considering a maximum round-trip input delay of $20 \mathrm{~ms}\left(\mathrm{PFC}_{c}\right)$ [8] and the $\mathrm{PFC}$ with taking into account an unknown time-varying input delay up to a maximum round-trip delay of $20 \mathrm{~ms}\left(\mathrm{PFC}_{v}\right)$ developed in Section 3.

In order to minimize the extra disturbance $d_{2}(n)$ in (13), the input of the system which represents the Closed Loop Transmit Power (CLTP) should be limited to the minimum possible variation. Therefore, in our simulations we bound the CLTP by $[-1 ; 1] \mathrm{dBm}[5]$.

Due to Theorem 3.1, $S$ is an invariant set if 
$-\frac{1}{2}<k_{1}<0$, and $k_{2}$ satisfies the following inequality

$$
k_{2} \geq \frac{(\varepsilon+2 M)^{2}\left(k_{1}+1\right)}{\left(2 k_{1}+1\right)^{2}} .
$$

However, equation (35) is only a sufficient condition. In order to consume less transmit power and have less interferences, we choose $k_{1}$ so that we have less possible received SINR. A numerical computation shows that the closer that $k_{1}$ is to -0.5 , the lower the level of the received SINR will be (see Figure 2). Accordingly, we choose $k_{1}=-0.49$ for our simulations. As already mentioned, equation (35) gives us a sufficient condition for choosing $k_{2}$, which for when $k_{1}=-0.49, k_{2}=636$. But by performing an offline adjustment we can find an optimal value for $k_{2}=300$.

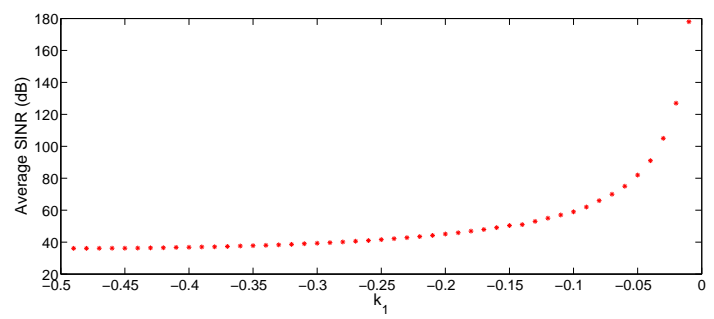

Fig. 2. Average of received SINR for different values of $k_{1}$ for $5 \mathrm{~s}$ simulation.

Figure 3(a) illustrates the evolution of the received SINR at the BS by using the TPC, the $\mathrm{PFC}_{c}$ and the $\mathrm{PFC}_{v}$ as the transmission power control scheme.

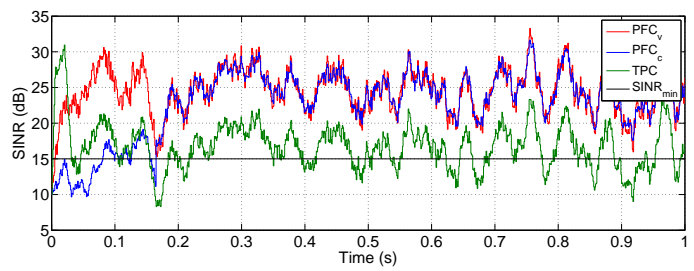

(a)

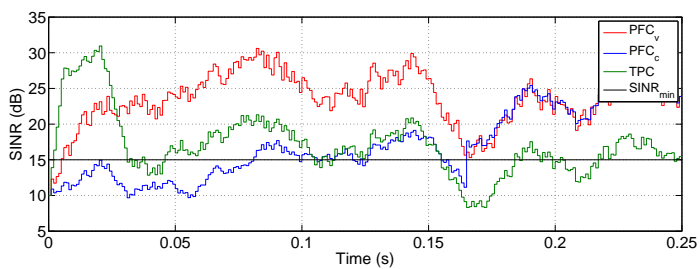

(b)

Fig. 3. (a) Received SINR evolution for 1s simulation. (b) Start-up comparison between the three ILPC schemes.

The TPC keeps the average SINR lower than the other two schemes but it can not maintain the connectivity for the whole communication duration. The $\mathrm{PFC}_{v}$ passes the $\mathrm{SINR}_{\text {min }}$ after a short period of time and stays above it to maintain the communication connectivity (see Figure $3(\mathrm{~b})$ ). The $\mathrm{PFC}_{c}$ has the same behavior than the
$\mathrm{PFC}_{v}$ with less oscillation compared to the $\mathrm{PFC}_{v}$ due to the extra disturbance $d_{2}(n)$. However, the $\mathrm{PFC}_{v}$ has a better performance in start-up compared to the $\mathrm{PFC}_{c}$ which due to the transmit power saturation can not maintain the SINR above the $\mathrm{SINR}_{\min }$ till $0.17 \mathrm{~s}$. As it has been explained in Section 2, the computed uplink transmit power $\mathrm{P}$ should not exceed the maximum MS transmit power $\mathrm{P}_{\max }$ which in LTE communication standard is $23 \mathrm{dBm}[1]$. As the $\mathrm{PFC}_{v}$ limits the input of the system, it does not exceed $\mathrm{P}_{\max }$. The $\mathrm{SINR}_{\min }$ is provided by the OLPC which also could be time-varying but with a lower rate than the ILPC (see Section 2), therefore this start-up lag can be an issue in this case.

Table 1

Performance comparison

\begin{tabular}{|l|c|c|c|}
\cline { 2 - 4 } \multicolumn{1}{c|}{} & $\mathrm{TPC}$ & $\mathrm{PFC}_{c}$ & $\mathrm{PFC}_{v}$ \\
\hline Execution Time (s) & 0.98 & 0.51 & 0.51 \\
\hline Average Received SINR (dB) & 16.7 & 24.7 & 24.9 \\
\hline $\begin{array}{l}\text { Time to pass the SINR } \\
\text { and }\end{array}$ & - & 0.17 & 0.01 \\
\hline
\end{tabular}

Finally, Table 1 compares performances of all three ILPC schemes for $10 \mathrm{~s}$ simulation. The $\mathrm{PFC}\left(\mathrm{PFC}_{v}\right.$ and $\left.\mathrm{PFC}_{c}\right)$ saves $48 \%$ of the computation time compared to the TPC due to the fact that the TPC algorithm performs a comparison for each ILPC sample time which is costly in computation time. However, the average received SINR of the $\mathrm{PFC}_{v}$ and the $\mathrm{PFC}_{c}$ are higher than the TPC which is the cost of ensuring the communication connectivity. Furthermore, the average received SINR for the $\mathrm{PFC}_{v}$ is slightly higher than the $\mathrm{PFC}_{c}$ while it passes the $\mathrm{SINR}_{\text {min }}$ and stays above it significantly faster than the $\mathrm{PFC}_{c}$ (see Figure 3(b) and Table 1). As it has been mentioned, the $\mathrm{SINR}_{\text {min }}$ in LTE communication standard is provided based on the chosen MCS by the AMC module in each OLPC sample time. In more realistic cases, considering the time-varying behavior of the transmission channel quality, the AMC module chooses different MCSs which leads to a time-varying SINR $_{\text {min }}$. In this case, the $\mathrm{PFC}_{v}$ is more reactive in the start-up compared to the other two ILPC schemes.

\section{Conclusion}

In this paper, we have developed the PFC based on the discrete-time Artstein transform for scalar discrete-time systems having a constraint on the state space variable in order to take into account an unknown bounded timevarying input delay. It is well suited for the ILPC of WCNs due to the constraint on the received SINR imposed by the communication standard and the delay caused by decision making and propagation time which is time-varying. Our simulations in LTE show that the PFC with taking into account an unknown bounded time-varying input delay $\left(\mathrm{PFC}_{v}\right)$ decreases the computation time and could ensure a better QoS by maintain- 
ing the communication connectivity compared to the TPC algorithm. Furthermore, it is significantly faster in maintaining the connectivity at start-up compared to the $\mathrm{PFC}_{c}$ which takes into account the maximum round trip delay as a constant input delay. The average received SINR of the $\mathrm{PFC}_{v}$ is higher than the other two methods. However we can not be sure that the $\mathrm{PFC}_{v}$ consumes more energy because the re-transmission procedure also must be taken into account for measuring the global energy consumption.

\section{References}

[1] 3GPP (3rd Generation Partnership Project). TS 36.1.101, Evolved Universal Terrestrial Radio Access (E-UTRA); User Equipment (UE) radio transmission and reception. Technical report, 2015.

[2] González A. Robust stabilization of linear discretetime systems with time-varying input delay. Automatica, 49(9):2919-2922, 2013.

[3] Sheth A. and Han R. Adaptive power control and selective radio activation for low-power infrastructure-mode 802.11 LANs. In 23rd IEEE International Conference on Distributed Computing Systems Workshops, pages 812-818, Providence, USA, 2003.

[4] Muhammad B. and Mohammed A. Performance evaluation of uplink closed loop power control for LTE system. In 70th IEEE Vehicular Technology Conference Fall, pages 1-5, Anchorage, Alaska, 2009.

[5] Cox C. An introduction to LTE: LTE, LTE-Advanced, SAE and $4 G$ mobile communications. John Wiley \& Sons, 2012.

[6] Castellanos C.U., Villa D.L., Rosa C., Pedersen K.l., Calabrese F.D., Michaelsen P.H., and Michel J. Performance of uplink fractional power control in UTRAN LTE. In IEEE Vehicular Technology Conference, pages 2517-2521, Singapore, 2008.

[7] Sung C.W. and Leung K.K. A generalized framework for distributed power control in wireless networks. IEEE Transactions on Information Theory, 51(7):2625-2635, 2005.

[8] Phan D., Moulay E., Coirault P., Launay F., and Combeau P. Potential feedback control for the power control in LTE. IEEE Transactions on Automatic Control, 60(9):2506-2511, 2015.

[9] Tse D. and Viswanath P. Fundamentals of wireless communication. Cambridge University Press, 2005.

[10] Gunnarsson F., Gustafsson F., and Blom J. Dynamical effects of time delays and time delay compensation in power controlled DS-CDMA. IEEE Journal on Selected Areas in Communications, 19(1):141-151, 2001.

[11] Khan F. LTE for $4 G$ mobile broadband: air interface technologies and performance. Cambridge University Press, 2009.

[12] Piro G., Grieco L.A., Boggia G., Capozzi F., and Camarda P. Simulating LTE cellular systems: an open-source framework. IEEE Transactions on Vehicular Technology, 60(2):498-513, 2011.

[13] Holma H. and Toskala A. LTE for UMTS-OFDMA and SCFDMA based radio access. John Wiley \& Sons, 2009.

[14] Feyzmahdavian H. R., Johansson M., and Charalambous T. Contractive interference functions and rates of convergence of distributed power control laws. IEEE Transactions on Wireless Communications, 11(12):4494-4502, 2012.
[15] Laiho J., Wacker A., and Novosad T. Radio network planning and optimisation for UMTS. John Wiley \& Sons, 2006.

[16] Luna-Rivera J.M. and Campos-Delgado D.U. Distributed power control with multiuser detection for asynchronous DSCDMA networks subject to time-delays. Telecommunication Systems, 52(4):2059-2069, 2013.

[17] Castro J.P. The UMTS network and radio access technology. John Wiley \& Sons, 2001.

[18] Song L. and Shen J. Evolved cellular network planning and optimization for UMTS and LTE. CRC Press, 2010.

[19] Yu L. and Gao F. Optimal guaranteed cost control of discretetime uncertain systems with both state and input delays. Journal of the Franklin Institute, 338(1):101-110, 2001.

[20] Chiang M., Hande P., Lan T., and Tan C.W. Power control in wireless cellular networks. Foundations and Trends in Networking, 2(4):381-533, 2008.

[21] Ogren P., Fiorelli E., and Leonard N. E. Cooperative control of mobile sensor networks: Adaptive gradient climbing in a distributed environment. IEEE Transactions on Automatic control, 49(8):1292-1302, 2004.

[22] Yates R.D. A framework for uplink power control in cellular radio systems. IEEE Journal on selected areas in communications, 13(7):1341-1347, 1995.

[23] Sesia S., Toufik I., and Baker M. LTE, The UMTS Long Term Evolution: From Theory to Practice. John Wiley \& Sons, 2009.

[24] Alpcan T., Başar T., Srikant R., and Altman E. CDMA uplink power control as a noncooperative game. Wireless Networks, 8(6):659-670, 2002.

[25] Rappaport T.S. Wireless Communications Principles and Practice (2nd Edition). Prentice Hall, 2001.

[26] Chen W.H., Guan Z.H., and Lu X. Delay-dependent guaranteed cost control for uncertain discrete-time systems with both state and input delays. Journal of the Franklin Institute, 341(5):419-430, 2004.

[27] Li X., Fang Q., and Shi L. A effective SINR link to system mapping method for CQI feedback in TD-LTE system. In IEEE 2nd International Conference on Computing, Control and Industrial Engineering (CCIE), volume 2, pages 208211, Wuhan, China, 2011.

[28] Xia Y., Liu G.P., Shi P., Chen J, Rees D., and Liang J. Sliding mode control of uncertain linear discrete time systems with input delay. IET Control Theory $\&$ Applications, 1(4):1169$1175,2007$. 